\title{
Compact cosmic ray detector for unattended atmospheric ionisation monitoring
}

Article

Accepted Version

Alpin, K.L. and Harrison, R.G. (2010) Compact cosmic ray detector for unattended atmospheric ionisation monitoring. Review of Scientific Instruments, 81 (12). 124501. ISSN 00346748 doi: https://doi.org/10.1063/1.3514986 Available at https://centaur.reading.ac.uk/17756/

It is advisable to refer to the publisher's version if you intend to cite from the work. See Guidance on citing.

Published version at: http://rsi.aip.org/resource/1/rsinak/v81/i12/p124501_s1

To link to this article DOI: http://dx.doi.org/10.1063/1.3514986

Publisher: AIP

Publisher statement: Copyright 2010 American Institute of Physics. This article may be downloaded for personal use only. Any other use requires prior permission of the author and the American Institute of Physics. The following article appeared in Review of Scientific Instruments, 81 (12). 124501 and may be found at 10.1063/1.3514986.

All outputs in CentAUR are protected by Intellectual Property Rights law, including copyright law. Copyright and IPR is retained by the creators or other copyright holders. Terms and conditions for use of this material are defined in the End User Agreement. 


\section{CentAUR}

Central Archive at the University of Reading

Reading's research outputs online 


\title{
Compact cosmic ray detector for unattended atmospheric ionisation monitoring
}

K.L. Aplin ${ }^{(1)}$ and R.G. Harrison ${ }^{(2)}$

1. University of Oxford, Department of Physics, Denys Wilkinson Building, Keble Road, Oxford OX1 3RH UK (corresponding author: k.aplin1@physics.ox.ac.uk)

2. University of Reading, Meteorology Department, PO Box 243, Earley Gate, Reading RG6 6BB UK

\begin{abstract}
Two vertical cosmic ray telescopes for atmospheric cosmic ray ionisation event detection are compared. Counter A, designed for low power remote use, was deployed in the Welsh mountains; its event rate increased with altitude as expected from atmospheric cosmic ray absorption. Independently, Counter B's event rate was found to vary with incoming particle acceptance angle. Simultaneous co-located comparison of both telescopes exposed to atmospheric ionisation showed a linear relationship between their event rates.
\end{abstract}

Keywords: Coincidence counter; Geiger-Müller tube; cosmic ray telescope 


\section{Introduction}

Ion generation in the atmosphere is now actively investigated because of its possible role in particle formation ${ }^{1}$ and cloud processes ${ }^{2}$, which may have a small effect on climate ${ }^{3}$. Related atmospheric experiments therefore require techniques that allow ionisation or cosmic ray measurements to be combined with conventional meteorological measurements. Direct measurement of surface atmospheric ions ${ }^{4}$ provides one approach, but the detection of cosmic ray ionisation events yields more information relevant to the effects of ionisation in the atmosphere well above the surface.

A long-established principle employed for cosmic ray detection is by the simultaneous triggering of two co-located Geiger-Müller tubes by high-energy particles. Such a "cosmic ray telescope" ${ }^{5}$ (originally known as a "coincidence counter" because of the simultaneous triggering aspect) was first used in 1929 to detect energetic ionising particles formed from cosmic rays $^{6}$. Scintillator techniques are now much more common in modern particle physics, but they have size and power requirements which limit their suitability for unattended operation in atmospheric fieldwork ${ }^{7}$. Consequently this paper investigates the cosmic ray telescope technique for long-term, low power unattended measurements of atmospheric ionisation. Because cosmic ray telescope measurements indicate the instant when a high-energy ionising particle has passed through the column of air above the detector, the telescope can be combined with other remote low power meteorological sensors to "trigger" measurements associated with the atmospheric ionisation effects. A further advantage is that, by monitoring the output of the individual Geiger-Müller tubes as 
well as the coincidence event times, the telescope can determine a variety of atmospheric ionisation sources, as the contribution from lower-energy cosmic ray electrons is small at the surface ${ }^{8}$. Cosmic ray telescopes can also be used effectively in education ${ }^{9}$, as they build on the familiar Geiger-Müller tubes to provide a simple and inexpensive way to demonstrate the properties of energetic particles.

Here, two compact cosmic ray telescopes (" $A$ " and " $B$ ") intended for long-term monitoring of atmospheric ionisation are investigated and compared. The telescopes employ a pair of Geiger-Müller (G-M) tubes in which simultaneous ionisation events are detected. Telescope A employs two ZP1442 G-M tubes, principally sensitive to $\beta$ and $\gamma$ radiation, and is intended for unattended, battery-powered operation at a remote site. Telescope B uses two ZP1410 G-M tubes, which are directly sensitive to atmospheric ionisation ${ }^{10}$ as they respond to $\alpha, \beta$ and $\gamma$ radiation.

Section 2 describes the telescopes' design considerations and section 3 presents independent tests: the telescope count rate variation with altitude, and variations of horizontal and vertical separation of the individual G-M tubes. Section 4 describes their direct comparison in atmospheric conditions.

\section{Principle of operation}

The telescopes operate in a similar way, each employing a pair of G-M tubes to detect highenergy events. Both devices are vertical telescopes, with, in both cases, their pairs of G-M tubes mounted with their long axes horizontal, as shown schematically in Figure 1 (a) and 
(b). The tubes require bias voltages of $\sim 500 \mathrm{~V}$ (which can vary within the "plateau" range of the tube's voltage-count response), supplied from independent low power high voltage supplies. The bias voltages were obtained using a standard electronics module ${ }^{11}$, which was based on a low voltage (12 V) CMOS square wave oscillator. The oscillator drove a miniature transformer and Cockcroft-Walton multiplier stages to nominally $500 \mathrm{~V}$, charging a $33 \mathrm{nF}$ smoothing capacitor. A voltage regulator was added to telescope A's bias supply, which, as for a previous low power G-M tube bias supply ${ }^{12}$, used a comparator to monitor the high voltage supply through a potential divider, activating the oscillator only when the smoothing capacitor's voltage fell below the G-M tube's plateau voltage. The bias supply for telescope B was unregulated, but the oscillator operated continuously.

Figure 1 (c) illustrates the signal processing employed. Pulses generated by the G-M tubes are used to trigger a monostable, which stretches them to $50 \mu \mathrm{s}$ (counter A) and $30 \mu \mathrm{s}$ (counter B). The stretched pulses are presented to an AND gate, the output of which goes high when G-M tubes pulses are present simultaneously within a detection window determined by the monostable pulse width. For counter A, the final AND gate "coincidence" pulse was counted directly using a Campbell data logger. In counter B, further pulse stretching was applied to yield a longer coincidence pulse of $\sim 1 s$, which was recorded using a digital counter, monitored every $5 \mathrm{~min}$. From each system an equivalent hourly coincidence count rate was determined, and the individual Geiger count rates recorded..

Both types of G-M tube used typically recorded a count rate of $\sim 10 / \mathrm{min}$ from radioactive decays. This background radioactivity, emitted from the soil, causes most of the 
atmospheric ionisation close to the ground ${ }^{13}$. It is therefore possible that some recorded coincidences will be "false", i.e. caused by the two individual G-M tubes being triggered close to simultaneously by different radioactive particles, rather than an energetic cosmic ray passing through both Geigers almost instantaneously. This rate is readily estimated ${ }^{6}$ as negligible $(<0.01 / \mathrm{hr})$ for both counters.

\section{Tests of the two coincidence counters}

(i) Telescope A

Telescope A was tested in a variety of ways, by attenuating the incoming particle flux, physically separating the detector's G-M tubes, and operating it in the atmosphere at different heights on a mountain. The usual operating configuration for counter $A$ was with the two G-M tubes stacked vertically, 63mm apart, each within separate IP68 sealed boxes which also contained their bias supplies, to form a vertical telescope. The size of the combined IP68 enclosures was $135 \times 137 \times 80 \mathrm{~mm}$, connected to an additional small IP68 sealed box ( $40 \times 40 \times 20 \mathrm{~mm})$ containing the coincidence circuitry.

Placing counter A immediately below $10 \mathrm{~cm}$ of lead made no difference to the coincidence rate, indicating, from the stopping energy ${ }^{14}$, that coincidences are only caused by particles with energies $>\sim 400 \mathrm{MeV}$. At the surface and in the lower atmosphere, almost all such energetic particles are expected to be muons (mean energy $4 \mathrm{GeV}^{14}$ ). Muons form by pion decay following primary cosmic ray collisions with molecules in the upper troposphere or lower stratosphere, and cause most of the cosmic ray ionisation in the troposphere ${ }^{15}$. 
In a second laboratory experiment, the coincidence rate was measured following horizontal displacement of the G-M tubes up to a separation of $1 \mathrm{~m}$. This displacement reduces the coincidence rate, since higher energy primary particles are needed to cause wide area events, known as "air showers". The fall-off of count rate with horizontal separation (the decoherence curve), follows a $x^{m}$ power law, for which $m$ has been previously observed as 0.1 to $-0.5^{16},-1{ }^{17}$, and $-2{ }^{9}$ Error! Bookmark not defined.. Results for Counter A gave $m=-$ $0.9 \pm 0.1$, in the middle of the expected range.

An atmospheric test of Counter A was to measure the change in coincidence rate with altitude, as atmospheric cosmic ray absorption falls off with height. To provide a large change in altitude, Counter A was taken to Mount Snowdon in Wales, height 1085m. During these experiments undertaken in 2005 , the coincidence rate was recorded at the base of the mountain, $128 \mathrm{~m}$, and then at the summit soon afterwards (Table 1 ). The experiment was repeated in 2009 with a common low-altitude site but using a different mountain nearby, Marchlyn Mawr (660m) for the upper altitude comparison. Both experiments showed a statistically significant increase in count rate at the mountain summit. The fractional increase in count rate was 1.17 for the $532 \mathrm{~m}$ altitude change in 2009 , compared to 1.46 for the $957 \mathrm{~m}$ altitude change for the 2005 measurements. The difference in the lowaltitude count rate between 2005 and 2009 may just arise from experimental variability; there is also the likelihood that the deep solar minimum in 2009 was permitting more cosmic rays to reach the lower altitude counter position. Background radioactivity was the 
same at all three locations, hence the false coincidence rate was not expected to contribute variability.

Counter A's responses to separation and altitude investigations are consistent with the variations expected from ionising cosmic ray particles.

\section{(ii) Telescope B}

Increasing the separation between two G-M tubes in a vertical telescope reduces the coincidence rate because of the change in acceptance angle defining the trajectory of a particle passing through both G-M tubes. This provides a further test of telescope operation. For two identical G-M tubes with their long axes $L$ aligned horizontally, the acceptance half angle $\alpha$ to the vertical within which high energy particles will pass through both tubes is related to their vertical separation $\mathrm{x}$ by $\tan \alpha=\mathrm{L} / \mathrm{x}$.

To investigate the vertical displacement effect on coincidence rate, the two enclosed G-M tubes of counter B were separated vertically and the coincidence rates averaged over several days at each separation. Although for the limiting case of $L / x=1$ the acceptance angle $\alpha=45^{\circ}$, the minimum separation in practice is limited by enclosures and mountings to $x / L$ 5, i.e. to $\alpha=9.8^{\circ}$. Figure 2 shows the reduction in coincidence rates obtained with increasing vertical separation. At a fixed distance above the Geigers, the effective horizontal capture area is proportional to $(\tan \alpha)^{2}$, i.e. proportional to $x^{-2}$. For the $\log -\log$ relationship in figure 2 , a fitted line of form $y=x^{n}$ gives $n=-2.2 \pm 0.7$, consistent with an inverse square law. 
During the two weeks of the separation experiments, the mean individual G-M tube count rates remained at $12 / \mathrm{min}$ which was independent of their position, hence the effect of separation on coincidence rate effect is not attributable to changes in the tube count rates.

\section{Atmospheric comparison of both counters}

A direct comparison between the counters in atmospheric conditions was made during an experiment carried out over four days at Reading University Atmospheric Observatory in the southern UK during a comprehensive atmospheric electricity experiment in May $2005^{18}$. Both instruments were independently powered and mounted outdoors within $1 \mathrm{~m}$ of each other. As the hourly count rates were obtained with separate logging systems, to allow comparison the timestamps of the counter B data were interpolated onto those of counter A using a cubic spline. The stochastic nature of the cosmic ray events precludes short term variations in the time series being closely correlated, but hour to day scale variations in muon production are modulated by pressure and upper atmosphere temperature changes $^{19}$, hence similar variations are expected on these timescales.

Figure 3 presents the time series for both instruments, and the relationship between them. The time series share similar features, and following interpolation onto a common time axis, the gradient of a line fitted by regression to the data is 1.04 . The mean count rates are similar, as the two counters employ similar geometry and dimensions of tubes and enclosures. The differences in the energy sensitivity of the different G-M tubes is not an important factor, as the energy of the coincidence-causing particles is always far greater than the sensitivity thresholds of the different tubes. 


\section{Summary}

Both telescope systems independently show appropriate responses under horizontal (Counter A) and vertical (Counter B) separation of their G-M tubes. Counter A's decoherence curve exponent is comparable with previous measurements, and its mean count rate responds quantitatively as expected for cosmic ray variations both with altitude and the solar cycle. Data from the combination of the two counters provides further confidence that the two devices are behaving consistently.

Experience with these telescope instruments in mountain regions over several years shows that this type of compact cosmic ray counter is suitable for long-term unattended monitoring of atmospheric ionisation.

\section{Acknowledgements}

Dr J. H. Williams assisted with the Welsh mountain site measurements and Dr R.J. Wilding and A.G. Lomas with the counter comparison. J.G. Firth designed and constructed the additional circuitry for counter A, and S.R. Tames constructed counter B. KLA acknowledges support of the UK Natural Environment Research Council (NERC) (NER/M/S/2003/00062) and Science and Technology Facilities Council (PP/E509065/1); the Reading work was supported by NERC (NERC/T/S/2002/00150). 
Table 1 Summary of coincidence rates measured using counter $A$ at three altitudes in Wales in 2005 and 2009.

Table 1

\begin{tabular}{|c|c|c|c|c|}
\hline & \multicolumn{2}{|r|}{2005} & \multicolumn{2}{|c|}{2009} \\
\hline $\begin{array}{l}\text { Location } \\
\text { (height } \\
\text { above sea } \\
\text { level) }\end{array}$ & $\begin{array}{l}\text { Date and } \\
\text { data } \\
\text { obtained }\end{array}$ & $\begin{array}{c}\text { Mean coincidence } \\
\text { rate (counts/hour) } \\
\text { (uncertainty one } \\
\text { standard error) }\end{array}$ & $\begin{array}{l}\text { Date and data } \\
\text { obtained }\end{array}$ & $\begin{array}{l}\text { Mean coincidence } \\
\text { rate (counts/ hour) } \\
\text { (uncertainty one } \\
\text { standard error) }\end{array}$ \\
\hline $\begin{array}{l}\text { Snowdon } \\
\text { Summit } \\
\text { (1085m) }\end{array}$ & $\begin{array}{l}26^{\text {th }} \text { July }-4^{\text {th }} \\
\text { August (days } \\
207-216 \\
\text { (1285 10min } \\
\text { totals) }\end{array}$ & $15.1 \pm 0.3$ & & \\
\hline $\begin{array}{l}\text { Marchlyn } \\
\text { Mawr } \\
(660 \mathrm{~m}) \\
\text { (7.6 km } \\
\text { north-north- } \\
\text { east of } \\
\text { Snowdon) }\end{array}$ & & & $\begin{array}{l}28^{\text {th }} \text { July }-25^{\text {th }} \\
\text { November (days } \\
209-329) \\
(32,6215 \text { min } \\
\text { totals) }\end{array}$ & $14.5 \pm 0.1$ \\
\hline $\begin{array}{l}\text { Llanberis } \\
(120 \mathrm{~m})\end{array}$ & $\begin{array}{l}25^{\text {th }}-26^{\text {th }} \text { July } \\
\text { (days 206- } \\
207 \text { ) } \\
\text { (81 10min } \\
\text { totals) }\end{array}$ & $10.3 \pm 1.0$ & $\begin{array}{l}13^{\text {th }}-14^{\text {th }} \text { July } \\
\text { (days } 194-195) \\
\text { (165 } 5 \text { min } \\
\text { totals) }\end{array}$ & $12.4 \pm 1.0$ \\
\hline
\end{tabular}




\section{Figure captions}

Figure 1 Conceptual arrangement of the Geiger-Müller (GM) tubes in the two vertical Geiger telescopes A and B. All the GM tubes are cylindrical, with their long axes (length L) aligned horizontally. The tubes in each pair are separated a vertical distance $x$. The tube dimensions are different in telescope $A$ and telescope $B$, and their approximate arrangement is shown in (a) for telescope A and (b) for telescope B. (c) summarises the tubes' signal conditioning. Individual pulses from each tube are stretched and identified as simultaneous by an AND gate, to generate a "coincidence" pulse when both GM tubes trigger simultaneously. The individual count rates of the two tubes can be monitored at G1 and G2.

Figure 2 Coincidence rate for different vertical separation of the Geiger-Muller tube pair comprising telescope $B$, with the upper tube kept at $1 \mathrm{~m}$ above the surface and the other at different positions vertically below it. The tube separation is given in multiples of the long axis $L$ of the tube $(L=4 \mathrm{~cm})$, which was aligned horizontally. Measurement durations were 3 days each for $0.5 \mathrm{~m}, 0.65 \mathrm{~m}$ and $0.75 \mathrm{~m}$ separations and 6 days for the $0.25 \mathrm{~m}$ separation. Error bars represent 2 standard errors on the mean count rate obtained, with a weighted power law line fitted by regression.

Figure 3 Comparison of two telescopes ( $\mathrm{A}$ and $\mathrm{B}$ ) in atmospheric air operated at the same site, logged independently. (a) Time series of hourly average coincidence rates (counter A thick black line and counter B thin red line). (b) Rates from both counters plotted against each other, following (cubic spline) interpolation of counter B values on to timestamps of counter A. A unit gradient line has been added (solid line), together with a line fitted to the data by regression (dashed line). 


\section{References}

${ }^{1}$ Duplissy, J., et al, Atmos. Chem. Phys., 10, 4, 1635-1647 (2010)

${ }^{2}$ Nicoll, K.A. and Harrison R.G., Geophys. Res. Lett.,37, L13802, doi:10.1029/2010GL043605 (2010)

${ }^{3}$ Carslaw, K., Nature, 460, 332-333. doi:10.1038/460332a (2009)

${ }^{4}$ Aplin, K.L., and Harrison, R.G., Rev Sci Instrum, 72, 8 3467-3469 (2001)

${ }^{5}$ Wilson J.G., Cosmic rays, Wykeham, London (1976)

${ }^{6}$ Bothe W. and Kolhörster W., Zeit. Phys., 56, $751-777$ (1929)

${ }^{7}$ Osprey S. et al, Geophys. Res. Lett., 36, L05809, doi:10.1029/2008GL036359 (2009)

${ }^{8}$ Bazilevskaya G.A. et al, Space Science Reviews 137, 149-173 doi: 10.1007/s11214-0089339-y (2008)

${ }^{9}$ Blanco F., Fichera F., La Rocca P., Librizzi F, Parasole O. and Riggi F., Phys. Educ. 41 204-207 (2006)

${ }^{10}$ Aplin K.L. and Harrison R.G., Rev. Sci. Instrum., 71, 8, 3037-3041 (2000)

${ }^{11}$ Velleman (K2645 kit) Legen Heirweg 33B-9890 GAVERE, Belgium

${ }^{12}$ Harrison R.G., Rev Sci Instrum 76, 126111 doi:10.1063/1.2149005 (2005)

${ }^{13}$ Chalmers J.A., Atmospheric electricity, 2nd edition, Pergamon, Oxford (1965)

${ }^{14}$ Hillas A.M., Cosmic rays, Pergamon, Oxford (1972)

${ }^{15}$ Hillas A.M. Cosmic rays in Kaye and Laby Tables of Physical and Chemical Constants, $16^{\text {th }}$

Edition (1995)

${ }^{16}$ Hodgson A.L., Proc. Phys. Soc. A, 64, 12, 384A (1951)

${ }^{17}$ Dunne P. and Miller A., Phys. Ed., 36, 322-326 (2001)

${ }^{18}$ Harrison R.G. and Aplin K.L., Atmos Res., 85, 199-208 (2007)

${ }^{19}$ Wolfendale A.W., Cosmic rays, Newnes Ltd, London (1963) 
(a) Telescope A

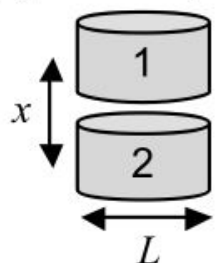

(b) Telescope B

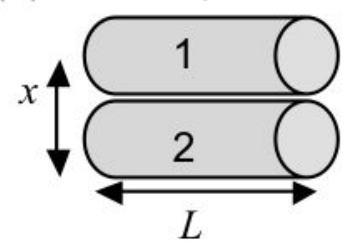

GM Tube 1

(c)

bias supply

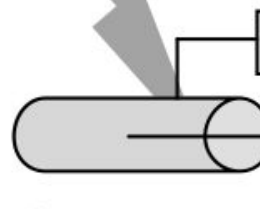

GM Tube 2
G1 output

\section{pulse stretcher}

bias supply pulse stretcher coincidence output

G2 output 


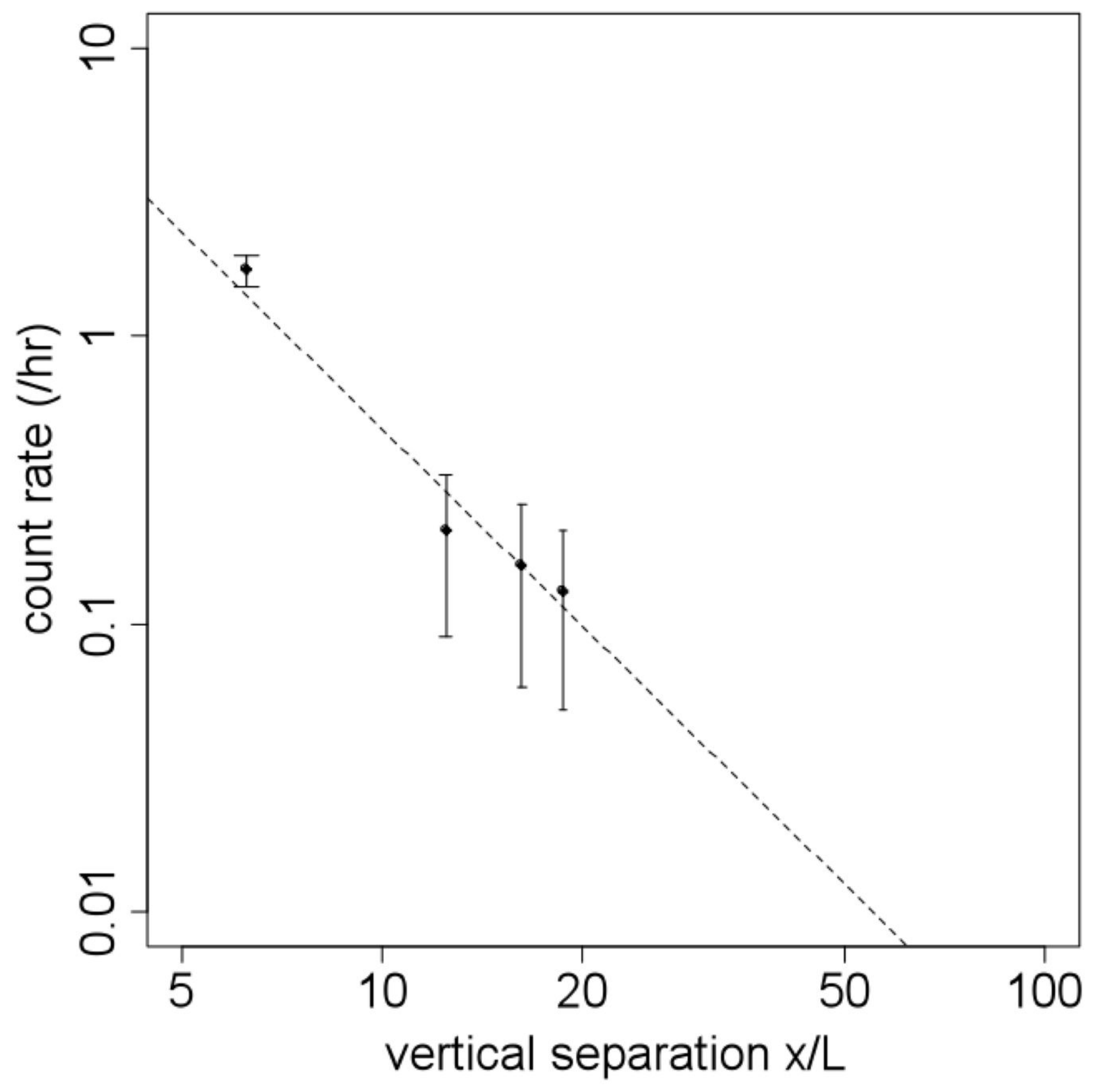


(a)

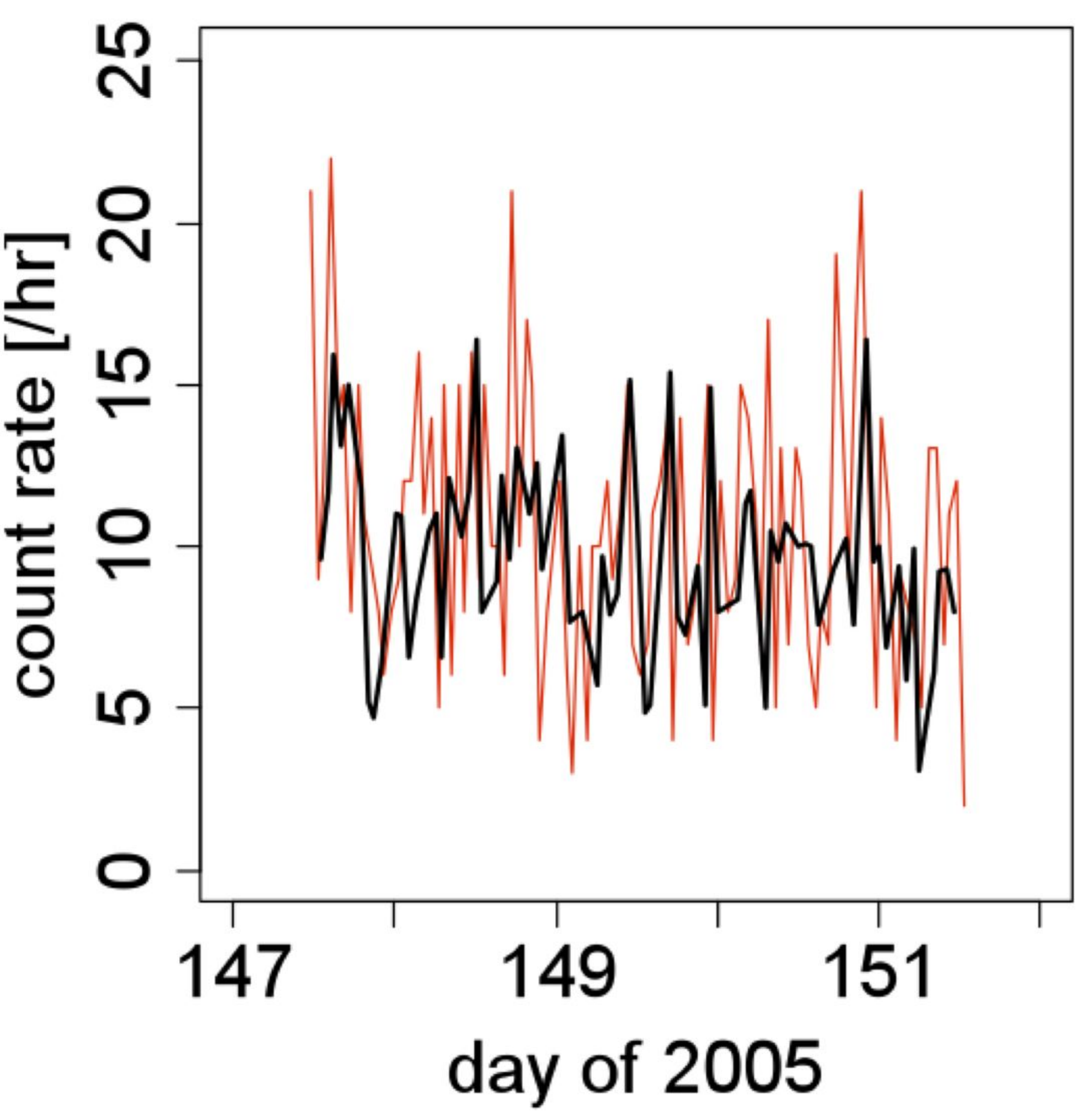

(b)

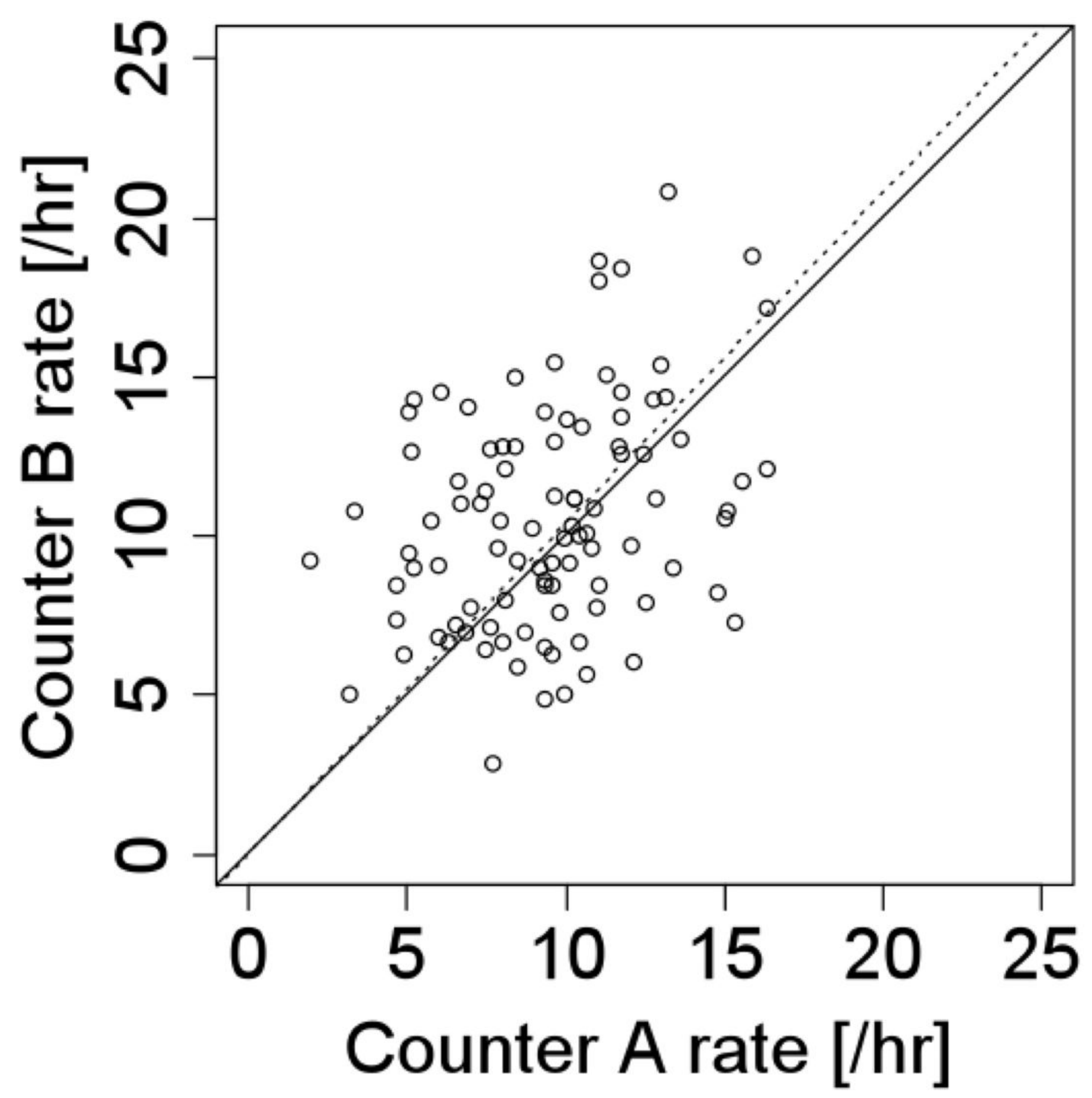

